\title{
Current Role of Atezolizumab Plus Bevacizumab Therapy in the Sequential Treatment of Unresectable Hepatocellular Carcinoma
}

\author{
Shohei Komatsu ( $\nabla$ komasho8@med.kobe-u.ac.jp ) \\ Kobe University Graduate School of Medicine \\ Yoshihiko Yano \\ Kobe University Graduate School of Medicine \\ Yoshimi Fujishima \\ Kobe Minimally Invasive Cancer Center \\ Jun Ishida
}

Kobe Minimally Invasive Cancer Center

Masahiro Kido

Kobe University Graduate School of Medicine

Kaori Kuramitsu

Kobe University Graduate School of Medicine

Atsushi Yamamoto

Kobe University Graduate School of Medicine

Tadahiro Goto

Kobe University Graduate School of Medicine

Hiroaki Yanagimoto

Kobe University Graduate School of Medicine

Hirochika Toyama

Kobe University Graduate School of Medicine

Yoshihide Ueda

Kobe University Graduate School of Medicine

\section{Yuzo Kodama}

Kobe University Graduate School of Medicine

\section{Takumi Fukumoto}

Kobe University Graduate School of Medicine

\section{Research Article}

Keywords: Hepatocellular carcinoma, Atezolizumab plus bevacizumab, Albumin-bilirubin grade, Lenvatinib, Treatment sequence 
Posted Date: October 8th, 2021

DOl: https://doi.org/10.21203/rs.3.rs-907325/v1

License: (c) (i) This work is licensed under a Creative Commons Attribution 4.0 International License. Read Full License

Version of Record: A version of this preprint was published at Anticancer Research on February 26th, 2022. See the published version at https://doi.org/10.21873/anticanres.15610. 


\section{Abstract \\ Background}

Atezolizumab plus bevacizumab therapy is the new standard treatment option for advanced hepatocellular carcinoma (HCC). The clinical details and sequential course after atezolizumab plus bevacizumab therapy remain to be determined.

\section{Methods}

From October 2020 to June 2021, 34 consecutive patients who received atezolizumab plus bevacizumab therapy were evaluated. Their clinical outcomes were assessed according to liver function classified by modified albumin-bilirubin (ALBI) grade 1 and $2 a(1 / 2 a)$ versus $2 b$ and treatment line (first-line versus second- or later-line). Furthermore, the treatment sequence after atezolizumab plus bevacizumab therapy was also assessed.

\section{Results}

The objective response and disease control rates were $15.6 \%$ and $93.8 \%$, respectively. The median proportions of ALBI scores at 1, 2, and 3 months relative to the baseline scores were $0.94,0.97$, and 0.93 , respectively. The median proportions of a-fetoprotein (AFP) scores at 1, 2, and 3 months relative to the baseline scores were $0.98,1.12$, and 1.83 , respectively. There were no significant differences in the changes in the proportions of AFP and ALBI scores according to both liver function and treatment line. Twelve patients were administered lenvatinib treatment after the failure of atezolizumab plus bevacizumab therapy. The proportions of AFP and ALBI scores at 1 month relative to the baseline scores were 0.55 and 0.81 , respectively.

\section{Conclusions}

Atezolizumab plus bevacizumab therapy can be administered effectively for advanced HCC irrespective of patients' liver function and treatment line. Lenvatinib administration after atezolizumab plus bevacizumab therapy can be effective, although special attention should be paid to the deterioration of liver function.

\section{Introduction}

Hepatocellular carcinoma (HCC) is one of the most common malignancies, and a major causes of cancer-related deaths worldwide [1, 2]. Owing to the late symptom development, HCC progression is often diagnosed at an advanced stage, which prevents the application of locoregional therapies. 
Systemic therapy is the mainstay in the management of patients with intermediate- or advanced-stage HCC refractory to or unsuitable for being treated with locoregional treatments [3, 4]. Since its approval in 2009, sorafenib was the only molecular target agent (MTA) for the first-line systemic treatment of advanced HCC that demonstrated a survival benefit over placebo [5] until lenvatinib was approved in 2018 based on the demonstration of noninferiority of overall survival compared to that of sorafenib [6].

Immune checkpoint inhibitors (ICls) have been widely investigated for use in the management of unresectable HCC. Nivolumab and pembrolizumab, anti-programmed cell death protein 1 antibodies, showed promising outcomes in phase $1 / 2$ trials but failed to show intended endpoint in both first- and second-line settings in subsequent phase 3 clinical trials [7]. Meanwhile, the combination of atezolizumab, a programmed death-ligand 1 inhibitor, and bevacizumab, an anti-vascular endothelial growth factor (VEGF) antibody, has recently produced highly encouraging results as first-line therapy for unresectable HCC in the phase 3 IMbrave150 trial based on the promising results of a phase $1 \mathrm{~b}$ study [8, 9].

With the emergence of atezolizumab plus bevacizumab as a first-line treatment, the therapeutic landscape of chemotherapy for HCC has changed dramatically, and there is no doubt that atezolizumab plus bevacizumab therapy will be the first-line treatment for advanced HCC. While the feasibility and potential effectiveness of atezolizumab plus bevacizumab therapy in the acute phase have recently become evident [10-13], one of the concerns is the selection of the optimal sequence of regimens after failure with atezolizumab plus bevacizumab therapy. Hence, the aim of the present study was to assess the detailed clinical course and investigate the treatment sequence after atezolizumab plus bevacizumab therapy.

\section{Patients And Methods}

\section{Patient enrollment}

The data of patients with advanced HCC without indications for locoregional therapies who received atezolizumab plus bevacizumab therapy at Kobe University Hospital and Kobe Minimally Invasive Cancer Center between October 2020 and June 2021 were reviewed. The diagnosis of the patients' condition was based on histopathology findings or clinical presentation; increased levels of serum a-fetoprotein (AFP) and serum protein induced by vitamin $\mathrm{K}$ absence or antagonist II (PIVKAll) and presence of detectable tumors on imaging techniques including computed tomography (CT) and magnetic resonance imaging (MRI). Performance status was evaluated according to the Eastern Cooperative Oncology Group, and the Barcelona Clinic Liver Cancer staging system was used to identify tumor stage [14]. Child-Pugh classification, albumin-bilirubin (ALBI) grade [15], and modified ALBI (mALBI) [16] were utilized for the assessment of liver function. Patients who were determined to have ALBI grade 2 were divided into two subgroups (mALBI $2 a$ and $2 b$ ) using an ALBI score of -2.27 as the cutoff value [17]. This study was conducted in accordance with the tenets of the Declaration of Helsinki and approved by the ethics 
committee of Kobe University in 2020 (approval number B200286). All patients provided written informed consent.

\section{Eligibility and exclusion criteria}

The eligibility criteria were: (1) an Eastern Cooperative Oncology Group performance status of $\leq 2$ and (2) adequate hematologic, renal, and hepatic function. The exclusion criteria were as follows: (1) serious complications other than chronic hepatitis or liver cirrhosis; (2) refractory ascites for which minimal therapeutic efficacy has been noted; and (3) severe autoimmune disease. HCC patients who fulfilled the inclusion criteria, did not meet the exclusion criteria, and provided written informed consent for participation were enrolled.

\section{Treatment schedule and assessment}

All patients received $1200 \mathrm{mg}$ of atezolizumab plus $15 \mathrm{mg} / \mathrm{kg}$ of bevacizumab intravenously every 3 weeks. Tumors were assessed through CT or MRI, and the therapeutic response was determined according to a modified version of the Response Evaluation Criteria in Solid Tumors [18, 19]. Adverse events were assessed according to the National Cancer Institute Common Toxicity Criteria version 5.0. Treatment was discontinued when unacceptable or serious adverse events occurred or clinical tumor progression was noted.

Laboratory data including serum AFP and PIVKAll levels, and tumor response, were assessed every 6-8 weeks using dynamic CT and/or MRI. The short-term outcomes after atezolizumab plus bevacizumab therapy, including changes in laboratory data, radiological response, adverse events, and treatment course, were evaluated. Special attention was focused on those with the liver function classified as mALBI grade 1 and $2 a(1 / 2 a)$ versus $2 b$ and those who received atezolizumab plus bevacizumab therapy as first-line versus second- or later-line therapy.

\section{Statistical analyses}

Quantitative data are presented as mean \pm standard deviation values. Qualitative data are presented as absolute numbers and percentages. Categorical variables were compared using the chi-square and Fisher's exact tests when appropriate. The non-parametric Mann-Whitney $U$ test was used to compare continuous variables, and chi-square test was performed to compare categorical variables among the groups. A pvalue of $<0.05$ was considered significant. Statistical analyses were conducted using the JMP 14 statistical package (SAS Institute, Cary, NC, USA).

\section{Results}

\section{Patients' baseline characteristics}

The clinical characteristics of 34 patients included in the present study are shown in Table 1. The median age was 73 years (range, 45-82 years). Regarding the cause of the background liver disease, 10 and 9 
patients had hepatitis $B$ and hepatitis $C$ virus infections, respectively, and 15 patients had non-B, non-C hepatitis. Fifteen (44.1\%) and 19 (55.9\%) patients had Barcelona Clinic Liver Cancer classification stage $B$ and C disease, respectively. Thirty-two (94.1\%) and 2 (5.9\%) patients were, respectively, classified as Child-Pugh class A and B. Eleven (32.4\%) and 23 (67.6\%) patients were classified as ALBI grades 1 and 2 , respectively. Regarding classification based on the mALBI score, 20 patients (58.8\%) were categorized as mALBI 1 and $2 \mathrm{a}(1 / 2 \mathrm{a})$, and $14(41.2 \%)$ as $2 \mathrm{~b}$. Of 34 patients, $13(38.2 \%)$ had more than 10 intrahepatic tumors and 13 (38.2\%) had extrahepatic metastases (i.e., nodal involvement and/or distant metastases) before the atezolizumab plus bevacizumab therapy. Twenty-four patients $(70.6 \%)$ had a history of systemic chemotherapy, and the remaining 10 patients $(29.4 \%)$ had not received HCC chemotherapy previously.

\section{Changes in hepatic function and tumor markers}

The changes in the proportions of ALBI scores at 1,2, and 3 months ( $n=34,33$, and 26, respectively) relative to the baseline scores are shown in Figure 1a. The median proportions of ALBI scores at 1,2, and 3 months relative to the baseline scores were $0.93,0.97$, and 0.93 , respectively. ALBI scores deteriorated at 1 month, slightly improved at 2 months, and deteriorated again at 3 months after the introduction of atezolizumab plus bevacizumab therapy. The changes in the ALBI scores according to the mALBI grade $(1 / 2 a$ versus $2 b)$ are shown in Figure $1 b$. The same trend could be observed for improvements in liver function could be seen at 2 months for both groups. However, at 3 months after treatment, liver function improved and deteriorated relative to that at baseline in the $\mathrm{MALBI} 2 \mathrm{~b}$ group (median ALBI score at 3 months relative to baseline score: 1.02) and mALBI 1/2a group (median ALBI score at 3 months relative to baseline score: 0.92 ), respectively; the difference was not significant. There were no significant differences in the changes in ALBI scores based on the treatment line (first-line versus second- or laterline) (Figure. 1c).

The changes in the proportions of AFP scores at 1,2, and 3 months relative to the baseline scores are shown in Figure $2 a$ (only for cases with preoperative elevation: AFP $\geq 10$ [26/34]). The median proportions of AFP scores at 1,2 , and 3 months ( $n=26,22$, and 20 , respectively) relative to the baseline scores were $0.98,1.12$, and 1.83 , respectively. In 13 of $25(52 \%)$ patients, the AFP level decreased at 1 month compared to the baseline level. Of these 13 patients, 9 patients had evaluable levels at 3 months after the treatment, and in 2 of these patients, the AFP levels were higher than the corresponding baseline level at 3 months. In the remaining 7 patients, the AFP level gradually decreased for up to 3 months. Among the remaining 12 patients whose AFP level at 1 month was higher than that at baseline (proportion at 1 month relative to that at baseline: $\geq 1$ ), 3 patients showed a decreased AFP level at 2 months compared to that at 1 month, while the remaining 9 patients showed increasing AFP levels with passage of time. There were no patients whose AFP level started to decrease at 3 months. The changes in AFP level according to the liver function determined based on the mALBI grade (1/2a versus $2 b)$ and treatment line (first-line versus second- or later-line) are shown in Figures $2 \mathrm{~b}$ and $2 \mathrm{c}$. There were no significant differences in the changes in AFP score proportion according to both the liver function and treatment line. 


\section{Radiological evaluation and adverse events}

Among the 34 patients, 32 (94.1\%) could undergo radiological evaluation (Table 2). None achieved complete response, and 5, 25, and 2 patients showed partial response, stable disease, and progressive disease, respectively. The overall response rate (ORR) and disease control rate (DCR) were $15.6 \%$ and $93.7 \%$, respectively. The ORRs were $10.5 \%(2 / 19)$ and $23.0 \%(3 / 13)$ for patients in the mALBI $1 / 2 \mathrm{a}$ and $2 \mathrm{~b}$ group, respectively, and the corresponding DCRs were $89.5 \%$ (17/19) and 100\% (13/13). There were no significant differences in ORRs and DCRs between the groups ( $P=0.34$ and 0.14$)$. The ORRs were $33.3 \%$ $(3 / 9)$ and $8.7 \%(2 / 23)$ for patients who received atezolizumab plus bevacizumab as first-line treatment and those who received this treatment as a second- or later-line therapy, respectively. The corresponding DCRs were $77.8 \%(7 / 9)$ and $100 \%(23 / 23)$, respectively, $(p=0.10$ and 0.02$)$. The ORR tended to be higher and DCR was significantly lower for patients in first-line group than that of those second- or later-line group (Table 2).

The most frequent adverse events associated with atezolizumab plus bevacizumab therapy at any grade was proteinuria, followed by serum aspartate aminotransferase / alanine aminotransferase disorder, decreased serum platelet count, general fatigue, and thyroid dysfunction, in this order. The proportion of adverse events of grade 3 or higher was $17.6 \%(6 / 34)$ and included perianal abscess $(n=1)$, proteinuria $(n=1)$, HCC rupture $(n=1)$, esophageal varices $(n=1)$, hypertension $(n=1)$, and perforation of the small intestine $(n=1)$.

\section{Treatment course after atezolizumab plus bevacizumab therapy}

Of the 34 patients who received atezolizumab plus bevacizumab therapy, 17 continued the regimen. Five patients discontinued chemotherapy and received the best supportive care, and 12 progressed to receive the next line of therapy. Of these 12 patients, all received lenvatinib as the next-line treatment option, and their clinical details are described in Table 3 (flowchart in Supplementary Fig .1). Ten patients started lenvatinib treatment within 14 days, and the remaining 2 patients started lenvatinib 21 and 24 days after the final administration of atezolizumab plus bevacizumab therapy, respectively. Among the 12 patients who received lenvatinib after atezolizumab plus bevacizumab therapy, 9 received a rechallenge with lenvatinib, and the remaining 3 had not received lenvatinib treatment previously. The changes in serum AFP level (only in those with an elevated AFP level: AFP $\geq 10$ [8/12]) after lenvatinib introduction are shown in Fig. 3. All 8 patients who had elevated serum AFP levels before lenvatinib introduction showed a decrease in these levels in 1 month (Table 3 ). The median proportions of AFP levels at 1,2 , and 3 months relative to the baseline levels were 0.55 (0.31-0.94), 0.68 (0.56-3.07), and $0.98(0.70-2.90)$, respectively. The AFP level increased in all 8 patients as time passed, from 1 to 2 months and 2 to 3 months, respectively, and it did not decrease in any patient.

The changes in ALBI scores after lenvatinib administration following the failure of atezolizumab plus bevacizumab therapy are shown in Figure 4. The median ALBI score was -2.24 (ranging from -3.11 to -1.68) before lenvatinib administration and -1.94 (range from - 2.52 to -0.94) at 1 month after lenvatinib administration. Six of the 12 patients were classified into the mALBI grade 1/2a group before lenvatinib 
administration; however, 3 patients showed aggravation of liver function at 1 month, resulting in their classification into the $2 \mathrm{~b} / 3$ group. The ALBI score worsened in 11 of the 12 patients, and the median proportion of ALBI score at 1 month relative to the baseline score was 0.81 (range: 0.46 to 1.18).

\section{Discussion}

The chemotherapeutic landscape of HCC has recently changed markedly due to the availability of several drug options [20,21]. Furthermore, the application of atezolizumab plus bevacizumab therapy in HCC management has led to an impressive shift in the treatment paradigm of advanced HCC.

The present study assessed the efficacy and feasibility of atezolizumab plus bevacizumab therapy according to the liver function (mALBI grade) and treatment line (first- or second- or later-line), and the treatment sequence after atezolizumab plus bevacizumab therapy. The short-term outcomes with an ORR of $15.6 \%$ and a severe complication rate of $17.6 \%$ in the present study were almost comparable to those reported in the IMbrave 150 study and recently published real-world data [9-11].

In the entire cohort, the ALBI score worsened at 1 month, slightly improved at 2 months, and deteriorated again at 3 months after the introduction of atezolizumab plus bevacizumab therapy. The same trend has been described previously [10], thus this trend may be a specific feature of atezolizumab plus bevacizumab therapy. The changes in the ALBI score according to the MALBI grade showed the discrepancy between the mALBI $1 / 2 a$ and $2 b$ groups. The liver function was better at 3 months than at baseline in the mALBI $2 \mathrm{~b}$ group (median ALBI score at 3 months relative to that at baseline: 1.02), whereas it worsened in the mALBI 1/2a group (median ALBI score at 3 months relative to that at baseline: 0.92). The reason for this discrepancy remains unknown, and we cannot conclude whether this trend was only seen in our cohort or is a common feature of atezolizumab plus bevacizumab therapy. Although long-term observation will be required, liver dysfunction induced by atezolizumab plus bevacizumab therapy in patients with deteriorated liver function might have little influence.

Regarding the changes is serum AFP level during treatment, as shown in Figure $2 \mathrm{a}$, the levels decreased in $52 \%(13 / 25)$ of patients, and the remaining $48 \%(12 / 25)$ patients were non-responders in terms of the serum AFP level at 1 month. Of the 13 patients who were responders in terms of a decrease in the AFP level, 7 out of the 9 patients continued to show a decrease in the AFP level until at least 3 months after the initiation of atezolizumab plus bevacizumab therapy, indicating that the treatment response continued until 3 months in those who were responders in terms of the serum AFP level at 1 month. Of the remaining 12 patients who were non-responders in terms of the serum AFP levels at 1 month, 3 showed decreased AFP levels at 2 months compared to the levels at 1 month. However, the remaining 9 patients showed increasing AFP levels as time passed. No patient showed a decrease in the serum AFP level from 3 months after treatment. If the AFP level does not decrease until 2 months after the treatment, a decrease in serum AFP level may not be expected, and these findings can be an important index for predicting the treatment response and deciding whether atezolizumab plus bevacizumab therapy should be continued. 
There were no significant differences in changes in ALBI score and serum AFP level, and radiological response according to the liver function ( $\mathrm{mALBI} 1 / 2 \mathrm{a}$ versus $2 \mathrm{~b}$ ) and treatment line (first-line versus second- or later-line). These findings indicate that atezolizumab plus bevacizumab therapy can be effective irrespective of the liver function and treatment line.

The treatment sequence after atezolizumab plus bevacizumab therapy will become a major concern in future HCC treatment. The results of the $\mathrm{G} 030140$ phase $1 \mathrm{~b}$ study demonstrated the effectiveness of combination treatment with atezolizumab plus bevacizumab compared to that with atezolizumab alone [8] and clearly indicated the usefulness of combination treatment with ICls and MTAs. The anti-VEGF activity of MTAs, which could affect the anti-angiogenesis activity and reprogram the tumor microenvironment from immunosuppressive to immunostimulatory, resulted in an increased response to ICls. In this scenario, the combination of ICls and MTAs could exert a synergic effect on advanced HCC and even on those refractory to $\mathrm{ICl}$ monotherapy.

Lenvatinib administration after atezolizumab plus bevacizumab therapy is a quite reasonable sequence based on the above rationale. The antitumor effect of lenvatinib is theoretically stronger than that of bevacizumab; thus, it can exert an antitumor effect even in cases in which the disease is refractory to atezolizumab plus bevacizumab therapy. Aoki et al. reported the favorable outcomes of lenvatinib administration after the programmed cell death 1/programmed death ligand 1 antibody therapy in clinical settings [22]. Regarding the timing of treatment sequence, Kudo et al. suggested that administration of these MTAs after ICls during several months may be more effective [23]. In the present study, 12 patients received lenvatinib after atezolizumab plus bevacizumab therapy, and 10 of the 12 patients received lenvatinib within 14 days after the final administration of atezolizumab plus bevacizumab therapy. Of the 12 patients, 8 who had elevated AFP levels before the lenvatinib administration all showed a decrease in the levels at 1 month after lenvatinib administration. However, the AFP levels increased from the second month after lenvatinib administration in all cases, indicating the attenuated effect of the $\mathrm{ICl}$ as time passed. Although lenvatinib administration immediately after atezolizumab plus bevacizumab therapy may have some impact in terms of an antitumor effect, its impact on long-term effectiveness should be further explored.

The deterioration of liver function associated with lenvatinib administration after atezolizumab plus bevacizumab therapy is also an important issue to be explored. Liver function deteriorated in 11 of 12 patients as indicated by the ALBI score, and the median proportion of the ALBI score at 1 month relative to that at baseline was 0.81 . These observations might be a specific feature of these consecutive treatments, and caution should be exercised when applying these treatments because some patients show significantly deteriorated liver function.

Regarding the treatment options after atezolizumab plus bevacizumab therapy, lenvatinib administration is the absolute option for lenvatinib-naïve patients. In patients with conditions once refractory to lenvatinib, there should be a greater focus on drug selection. Rechallenge chemotherapy is an essential and established concept in the management of various malignancies [24-28]. Although, there has been 
almost no report regarding rechallenge chemotherapy for HCC, it may be an effective option in the setting of later-line chemotherapy. Indeed, in the present study, of the 12 patients administered lenvatinib after atezolizumab plus bevacizumab therapy, 9 received rechallenges after lenvatinib failure. The changes in AFP levels (decrease) in these 9 patients were similar to those in lenvatinib-naïve patients, suggesting its effectiveness; nevertheless, further follow-up will be necessary.

The present study has several limitations. First, the study was a retrospective study with a small sample size. Second, the overall survival was not evaluated because of the short observation period. However, the study showed important short-term outcomes of atezolizumab plus bevacizumab therapy in patients with HCC. Thus, further investigation with long-term observation is warranted to confirm treatment effectiveness.

In conclusion, atezolizumab plus bevacizumab therapy is relatively safe and effective for the treatment of $\mathrm{HCC}$, irrespective of patients' liver function and treatment line. The treatment sequence after atezolizumab plus bevacizumab therapy will be an urgent issue in future clinical practice, and lenvatinib can be a useful treatment choice, although long-term observation is must with this approach.

\section{Abbreviations}

HCC: Hepatocellular carcinoma; MTA: Molecular target agent; ICl: Immune checkpoint inhibitor; VEGF: Vascular endothelial growth factor; AFP: a-fetoprotein; PIVKAll: Protein induced by vitamin K absence or antagonist II; CT: Computed tomography; MRI: magnetic resonance imaging; ALBI: Albumin-bilirubin; mALBI: Modified ALBI; ORR: Overall response rate; DCR: Disease control rate

\section{Declarations}

\section{Acknowledgments}

Not applicable.

\section{Authors' contributions}

Study conception and design: SK, YY, YF.

Acquisition of data: Jl, MK, KK, AY.

Analysis and interpretation of data: TG, HY, HT, YU, YK.

Drafting of manuscript: SK, TF.

All authors have read and approved the manuscript.

\section{Funding}


This research did not receive any specific grant from funding agencies in the public, commercial, or notfor-profit sectors.

\section{Availability of data and materials}

The datasets used and analyzed during the current study are available from the corresponding authors on reasonable request.

\section{Ethics approval and consent to participate}

This study was performed in line with the principles of the Declaration of Helsinki. This study was approved by the local ethics committee of Kobe University (approval number B200286). Informed consent was obtained from all subjects involved in the study.

\section{Consent for publication}

Not applicable.

\section{Competing interests}

The authors declare that they have no conflict of interest.

\section{Author details}

${ }^{1}$ Department of Surgery, Division of Hepato-Biliary-Pancreatic Surgery, Kobe University Graduate School of Medicine, Kobe, Hyogo, Japan. ${ }^{2}$ Department of Internal Medicine, Division of Gastroenterology, Kobe University Graduate School of Medicine, Kobe, Hyogo, Japan. ${ }^{3}$ Division of Medical Oncology, Kobe Minimally Invasive Cancer Center, Kobe, Japan. ${ }^{4}$ Division of Radiology, Kobe Minimally Invasive Cancer Center, Kobe, Japan

\section{References}

1. Ferlay J, Soerjomataram I, Dikshit R, Eser S, Mathers C, Rebelo M, et al. Cancer incidence and mortality worldwide: sources, methods and major patterns in GLOBOCAN 2012. Int J Cancer. 2015;136:E359-86.

2. European Association for the Study of the Liver. Electronic address eee, European Association for the Study of the L EASL Clinical Practice Guidelines: Management of hepatocellular carcinoma. J Hepatol. 2018;69:182-236.

3. Kloeckner R, Galle PR, Bruix J Local and Regional Therapies for Hepatocellular Carcinoma. Hepatology. 2021;73 Suppl 1:137-49. 
4. Llovet JM, Villanueva A, Marrero JA, Schwartz M, Meyer T, Galle PR, et al. Trial Design and Endpoints in Hepatocellular Carcinoma: AASLD Consensus Conference. Hepatology. 2021;73 Suppl 1:158-91.

5. Llovet JM, Ricci S, Mazzaferro V, Hilgard P, Gane E, Blanc JF, et al. Sorafenib in advanced hepatocellular carcinoma. N Engl J Med. 2008;359:378-90.

6. Kudo M, Finn RS, Qin S, Han KH, Ikeda K, Piscaglia F, et al. Lenvatinib versus sorafenib in first-line treatment of patients with unresectable hepatocellular carcinoma: a randomised phase 3 noninferiority trial. Lancet. 2018;391:1163-73.

7. Finn RS, Ryoo BY, Merle P, Kudo M, Bouattour M, Lim HY, et al. Pembrolizumab As Second-Line Therapy in Patients With Advanced Hepatocellular Carcinoma in KEYNOTE-240: A Randomized, Double-Blind, Phase III Trial. J Clin Oncol. 2020;38:193-202.

8. Lee MS, Ryoo BY, Hsu CH, Numata K, Stein S, Verret W, et al. Atezolizumab with or without bevacizumab in unresectable hepatocellular carcinoma (G030140): an open-label, multicentre, phase 1b study. Lancet Oncol. 2020;21:808-20.

9. Finn RS, Qin S, Ikeda M, Galle PR, Ducreux M, Kim TY, et al. Atezolizumab plus Bevacizumab in Unresectable Hepatocellular Carcinoma. N Engl J Med. 2020;382:1894-905.

10. Hiraoka A, Kumada T, Tada T, Hirooka M, Kariyama K, Tani J, et al. Atezolizumab plus bevacizumab treatment for unresectable hepatocellular carcinoma: Early clinical experience. Cancer Rep (Hoboken). 2021:e1464.

11. Yoo C, Kim JH, Ryu MH, Park SR, Lee D, Kim KM, et al. Clinical Outcomes with Multikinase Inhibitors after Progression on First-Line Atezolizumab plus Bevacizumab in Patients with Advanced Hepatocellular Carcinoma: A Multinational Multicenter Retrospective Study. Liver Cancer. 2021;10:107-14.

12. Iwamoto H, Shimose S, Noda Y, Shirono T, Niizeki T, Nakano M, et al. Initial Experience of Atezolizumab Plus Bevacizumab for Unresectable Hepatocellular Carcinoma in Real-World Clinical Practice. Cancers (Basel). 2021;13.

13. Kuzuya T, Ishigami M, Ito T, Ishizu Y, Honda T, Ishikawa T, et al. Initial Experience of Ramucirumab Treatment After Lenvatinib Failure for Patients With Advanced Hepatocellular Carcinoma. Anticancer Res. 2020;40:2089-93.

14. Llovet JM, Bruix J Novel advancements in the management of hepatocellular carcinoma in 2008. J Hepatol. 2008;48(Suppl. 1):S20-37.

15. Johnson PJ, Berhane S, Kagebayashi C, Satomura S, Teng M, Reeves HL, et al. Assessment of liver function in patients with hepatocellular carcinoma: a new evidence-based approach-the ALBI grade. J Clin Oncol. 2015;33:550-8.

16. Hiraoka A, Kumada T, Michitaka K, Toyoda H, Tada T, Ueki H, et al. Usefulness of albumin-bilirubin grade for evaluation of prognosis of 2584 Japanese patients with hepatocellular carcinoma. J Gastroenterol Hepatol. 2016;31:1031-6.

17. Hiraoka A, Michitaka K, Kumada T, Izumi N, Kadoya M, Kokudo N, et al. Validation and Potential of Albumin-Bilirubin Grade and Prognostication in a Nationwide Survey of 46,681 Hepatocellular 
Carcinoma Patients in Japan: The Need for a More Detailed Evaluation of Hepatic Function. Liver Cancer. 2017;6:325-36.

18. Eisenhauer EA, Therasse P, Bogaerts J, Schwartz LH, Sargent D, Ford R, et al. New response evaluation criteria in solid tumours: revised RECIST guideline (version 1.1). Eur J Cancer. 2009;45:228-47.

19. Lencioni R, Llovet JM Modified RECIST (mRECIST) assessment for hepatocellular carcinoma. Semin Liver Dis. 2010;30:52-60.

20. Zhu AX, Kang YK, Yen CJ, Finn RS, Galle PR, Llovet JM, et al. Ramucirumab after sorafenib in patients with advanced hepatocellular carcinoma and increased alpha-fetoprotein concentrations (REACH-2): a randomised, double-blind, placebo-controlled, phase 3 trial. Lancet Oncol. 2019;20:28296.

21. Abou-Alfa GK, Meyer T, Cheng AL, El-Khoueiry AB, Rimassa L, Ryoo BY, et al. Cabozantinib in Patients with Advanced and Progressing Hepatocellular Carcinoma. N Engl J Med. 2018;379:54-63.

22. Aoki T, Kudo M, Ueshima K, Morita M, Chishina H, Takita M, et al. Exploratory Analysis of Lenvatinib Therapy in Patients with Unresectable Hepatocellular Carcinoma Who Have Failed Prior PD-1/PD-L1 Checkpoint Blockade. Cancers (Basel). 2020;12.

23. Kudo M Sequential Therapy for Hepatocellular Carcinoma after Failure of Atezolizumab plus Bevacizumab Combination Therapy. Liver Cancer. 2021;10:85-93.

24. Kuczynski EA, Sargent DJ, Grothey A, Kerbel RS Drug rechallenge and treatment beyond progression-implications for drug resistance. Nat Rev Clin Oncol. 2013;10:571-87.

25. Felicetti F, Nervo A, Piovesan A, Berardelli R, Marchisio F, Gallo M, et al. Tyrosine kinase inhibitors rechallenge in solid tumors: a review of literature and a case description with lenvatinib in thyroid cancer. Expert Rev Anticancer Ther. 2017;17:1093-8.

26. Cremolini C, Rossini D, Dell'Aquila E, Lonardi S, Conca E, Del Re M, et al. Rechallenge for Patients With RAS and BRAF Wild-Type Metastatic Colorectal Cancer With Acquired Resistance to First-line Cetuximab and Irinotecan: A Phase 2 Single-Arm Clinical Trial. JAMA Oncol. 2019;5:343-50.

27. Agulnik M, Giel JL Understanding rechallenge and resistance in the tyrosine kinase inhibitor era: imatinib in gastrointestinal stromal tumor. Am J Clin Oncol. 2014;37:417-22.

28. Marotta V, Colao A, Faggiano A Complete disappearance of liver metastases in a patient with iodinerefractory differentiated thyroid cancer subjected to sorafenib re-challenge. Endocrine. 2015;50:8245.

\section{Tables}

Table 1 Patient characteristics of all patients 


\section{Characteristics}

Age, y, median (range)

Sex, male : female

ECOG PS, $0: 1$

Etiology, HBV : HCV : NBNC

BCLC classification, B : C

Child-Pugh class, A : B

AST, IU/L, median (range)

ALT, IU/L, median (range)

Total bilirubin, $\mathrm{mg} / \mathrm{dL}$, median (range)

Albumin, $\mathrm{g} / \mathrm{dL}$, median (range)

ALBI grade, $1: 2$

Modified ALBI grade, 1/2a : 2b

Prothrombin time, \%, median (range)

Platelet count $x 10^{9} / \mathrm{L}$, median (range)

AFP, ng/ml, median (range)

$$
\leq 10
$$

$10<$

PIVKAll, $\mathrm{mAU} / \mathrm{ml}$, median (range)

$<40$

$40 \leq$

Maximal intrahepatic tumor size, $\mathrm{mm}$, median (range)

Number of intrahepatic tumors, none : $<10: 10 \leq$

Vascular invasion of intrahepatic tumors, yes : no

Extrahepatic involvement, yes : no

History of systemic chemotherapy, yes : no

Treatment line of atezolizumab plus bevacizumab, 1st/2nd/3rd/4th/5th/6th

ECOG PS Eastern cooperative Oncology Group performance status, $H B V$ hepatitis B virus,
All patients

$(n=34)$

$73(45-82)$

$25: 9$

$18: 16$

$10: 9: 15$

$15: 19$

$32: 2$

38 (30-57)

30 (22-47)

0.8 (0.6-1.3)

$3.7(3.2-4.1)$

$11: 23$

$20: 14$

93.3 (83.4-102.9)

$12.9(9.9-16.4)$

257 (2-90770)

8

26

654 (15-72496)

6

28

28 (11-120)

$5: 16: 13$

$3: 31$

$13: 21$

$24: 10$

$10 / 12 / 6 / 3 / 2 / 1$ 
$H C V$ hepatitis $C$ virus, $N B N C$ non B non C, BCLC Barcelona Liver Clinic, $A S T$ aspartate aminotransferase, $A L T$ alanine aminotransferase, $A L B /$ albumin-bilirubin, $A F P$ alphafetoprotein, PIVKA/l protein induced by vitamin K absence or antagonist II

Table 2 Radiological response according to liver function and treatment line

\begin{tabular}{llllll} 
Radiological response & $\begin{array}{l}\text { All patients } \\
(\mathbf{n = 3 2})\end{array}$ & $\begin{array}{l}\text { mALBI 1/2a } \\
(\mathbf{n}=19)\end{array}$ & $\begin{array}{l}\text { mALBI 2b } \\
(\mathbf{n = 1 3 )}\end{array}$ & $\begin{array}{l}\text { First-line } \\
(\mathbf{n}=\mathbf{9})\end{array}$ & $\begin{array}{l}\text { Second- or later-line } \\
(\mathbf{n}=\mathbf{2 3})\end{array}$ \\
\hline Complete response & $0(0)$ & $0(0)$ & $0(0)$ & $0(0)$ & $0(0)$ \\
\hline Partial response & $5(15.6)$ & $2(10.5)$ & $3(23.0)$ & $3(33.3)$ & $2(8.7)$ \\
\hline Stable disease & $25(78.1)$ & $15(79.0)$ & $10(77.0)$ & $4(44.5)$ & $21(91.3)$ \\
\hline Progressive disease & $2(6.3)$ & $2(10.5)$ & $0(0)$ & $2(22.2)$ & 0 \\
\hline Overall response rate & $15.6 \%$ & $10.5 \%$ & $23.0 \%$ & $33.3 \%$ & $8.7 \%$ \\
\hline Disease control rate & $93.7 \%$ & $89.5 \%$ & $100.0 \%$ & $77.8 \%$ & $100.0 \%$
\end{tabular}

$A L B /$ albumin-bilirubin

Table 3 Clinical details of 12 patients who underwent lenvatinib administration after atezolizumab plus bevacizumab 


\begin{tabular}{|c|c|c|c|c|c|c|}
\hline Age & ALBI score & ALBI score & ALBI & AFP & AFP & AFP \\
\hline Sex & $\begin{array}{l}\text { (mALBI grade) } \\
\text { Before }\end{array}$ & $\begin{array}{l}\text { (mALBI grade) } 1 \\
\text { month }\end{array}$ & 1month/before & Before & $\begin{array}{l}1 \\
\text { month }\end{array}$ & $\begin{array}{l}1 \\
\text { month/before }\end{array}$ \\
\hline $\begin{array}{l}66 \\
M\end{array}$ & $-3.11(1)$ & $-2.31(2 a)$ & 0.74 & 3 & 2 & - \\
\hline $\begin{array}{l}63 \\
M\end{array}$ & $-2.74(1)$ & $-2.52(2 a)$ & 0.92 & 758 & 239 & 0.31 \\
\hline $\begin{array}{l}75 \\
M\end{array}$ & $-2.70(1)$ & $-2.32(2 a)$ & 0.86 & 4.4 & 5.3 & - \\
\hline $\begin{array}{l}72, \\
F\end{array}$ & $-2.62(1)$ & $-1.96(2 b)$ & 0.75 & 41419 & 32460 & 0.78 \\
\hline $\begin{array}{l}75 \\
M\end{array}$ & $-2.30(2 a)$ & $-1.91(2 b)$ & 0.83 & 16 & 15 & 0.94 \\
\hline $\begin{array}{l}48 \\
M\end{array}$ & $-2.25(2 a)$ & $-2.14(2 b)$ & 0.95 & 72834 & 37909 & 0.52 \\
\hline $\begin{array}{l}\text { 60, } \\
\mathrm{F}\end{array}$ & $-2.23(2 b)$ & $-1.03(3)$ & 0.46 & 213174 & 72756 & 0.34 \\
\hline $\begin{array}{l}53 \\
M\end{array}$ & $-1.88(2 b)$ & $-1.75(2 b)$ & 0.93 & 2050 & 1870 & 0.91 \\
\hline $\begin{array}{l}74, \\
M\end{array}$ & $-1.86(2 b)$ & $-2.19(2 b)$ & 1.18 & 29855 & 17107 & 0.57 \\
\hline $\begin{array}{l}66 \\
M\end{array}$ & $-1.79(2 b)$ & $-1.16(3)$ & 0.65 & 6 & 8 & - \\
\hline $\begin{array}{l}78, \\
M\end{array}$ & $-1.79(2 b)$ & $-1.68(2 b)$ & 0.94 & 3385 & 1052 & 0.31 \\
\hline $\begin{array}{l}72 \\
M\end{array}$ & $-1.68(2 b)$ & $-0.94(3)$ & 0.56 & 2 & 2 & - \\
\hline
\end{tabular}

$A L B /$ albumin-bilirubin, $A F P$ alpha-fetoprotein

Figures 
(a)

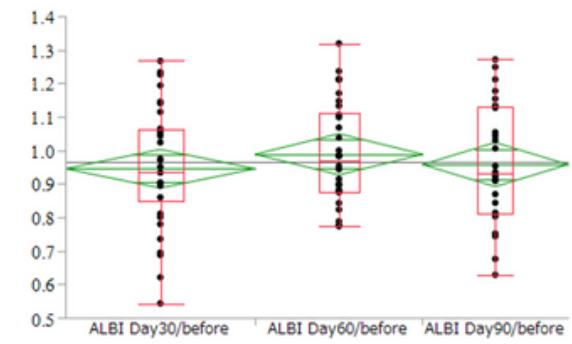

$\begin{array}{cccc}\text { Median } & 0.93 & 0.97 & 0.93 \\ \text { (range) } & (0.54 \sim 1.27) & (0.77 \sim 1.32) & (0.63 \sim 1.27)\end{array}$

(b)
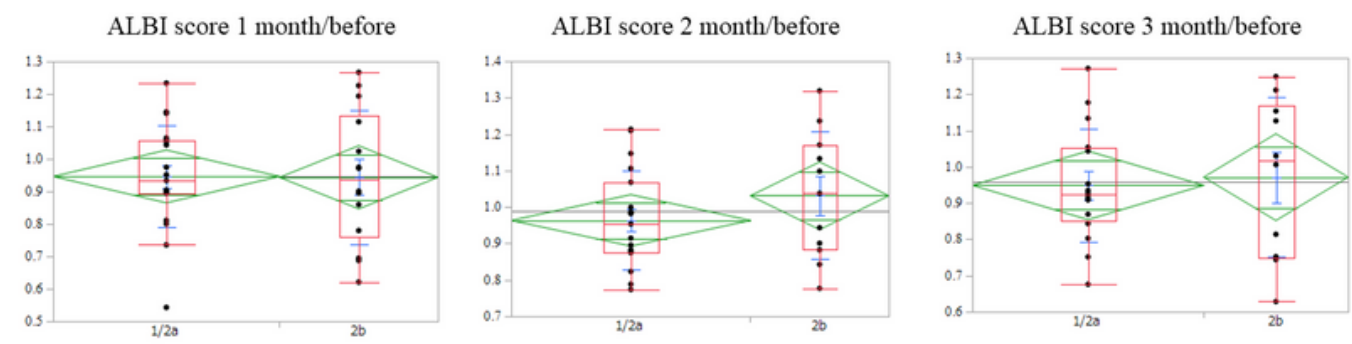

$1 / 2 \mathrm{a}: 0.93(0.54 \sim 1.23)$

$2 \mathrm{~b}: 0.94(0.62 \sim 1.27)$

$\mathrm{P}=0.9664$

$1 / 2 \mathrm{a}: 0.95(0.77 \sim 1.21)$

$2 \mathrm{~b}: 1.03(0.77 \sim 1.32)$

$\mathrm{P}=0.2450$

$1 / 2 \mathrm{a}: 0.92(0.68 \sim 1.27)$

$2 \mathrm{~b}: 1.02(0.63 \sim 1.25)$

$\mathrm{P}=0.7612$

(c)
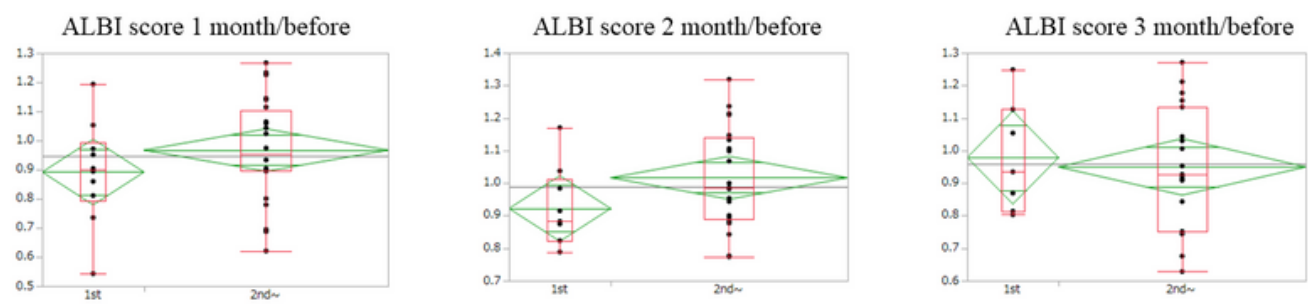

$$
\text { First line }
$$

$: 0.90(0.54 \sim 1.20)$

Second- or later-line : $0.95(0.62 \sim 1.27)$

$\mathrm{P}=0.2597$

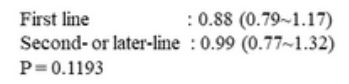
First line $\quad: 0.93(0.80 \sim 1.25)$
Second-or later-line $: 0.93(0.63 \sim 1.27)$
$P=0.7251$

\section{Figure 1}

(a) The proportions of ALBI scores at 1,2, and 3 months relative to the baseline scores in the entire cohort (b) The proportions of ALBI scores at 1, 2, and 3 months relative to the baseline scores according to modified ALBI grade 1/2a versus $2 b$. (c) The proportions of ALBI scores at 1, 2, and 3 months relative to the baseline scores according to the treatment line (first-line versus second- or later-line). ALBI, albuminbilirubin. 
(a)

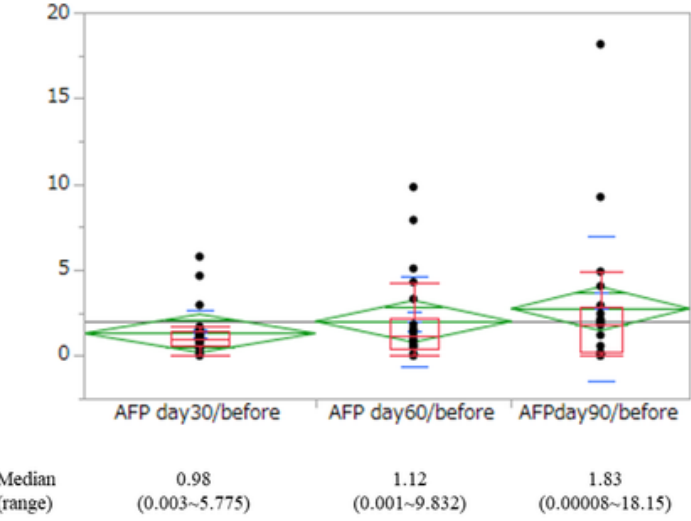

(b)
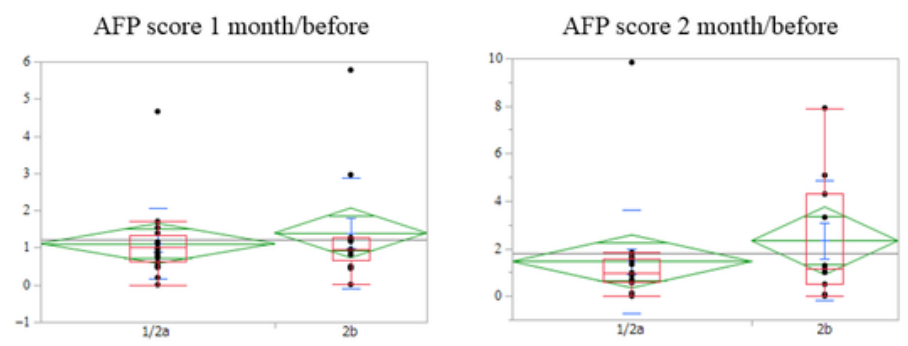

$1 / 2 \mathrm{a}: 1.00(0.003 \sim 4.66)$

$2 \mathrm{~b}: 0.95(0.01 \sim 5.78)$

$\mathrm{P}=0.5064$

$1 / 2 \mathrm{a}: 0.99(0.001 \sim 9.83)$
$2 \mathrm{~b}: 1.14(0.005 \sim 7.91)$
$\mathrm{P}=0.3312$

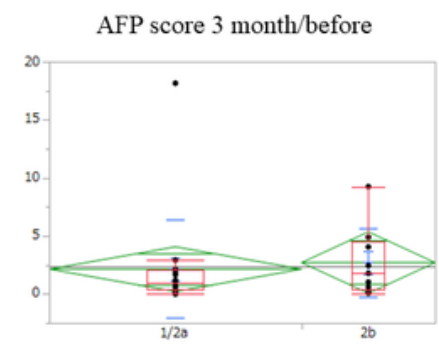

$1 / 2 \mathrm{a}: 1.00(0.00008 \sim 18.15)$

$2 \mathrm{~b}: 1.79(0.07 \sim 9.25)$

$\mathrm{P}=0.7246$

(c)
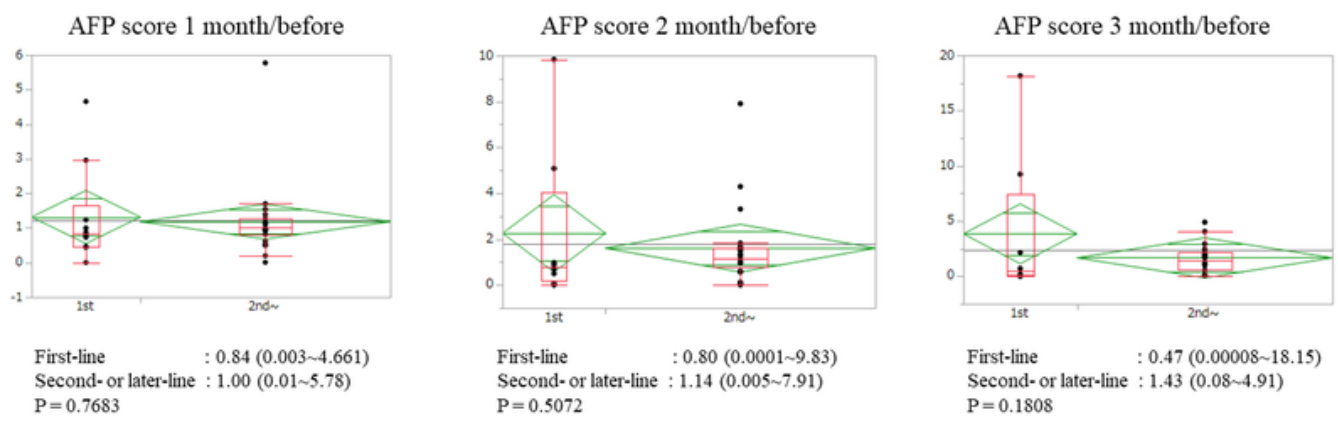

\section{Figure 2}

(a) The proportions of the a-fetoprotein level at 1, 2, and 3 months relative to the baseline level in the entire cohort (b) The proportions of the a-fetoprotein level at 1,2, and 3 months relative to the baseline level according to the modified ALBI grade $1 / 2$ a versus $2 b$. (c) The proportions of the a-fetoprotein level at 1,2 , and 3 months relative to the baseline level according to the treatment line (first-line versus secondor later-line). $\mathrm{ALBI}$, albumin-bilirubin. 


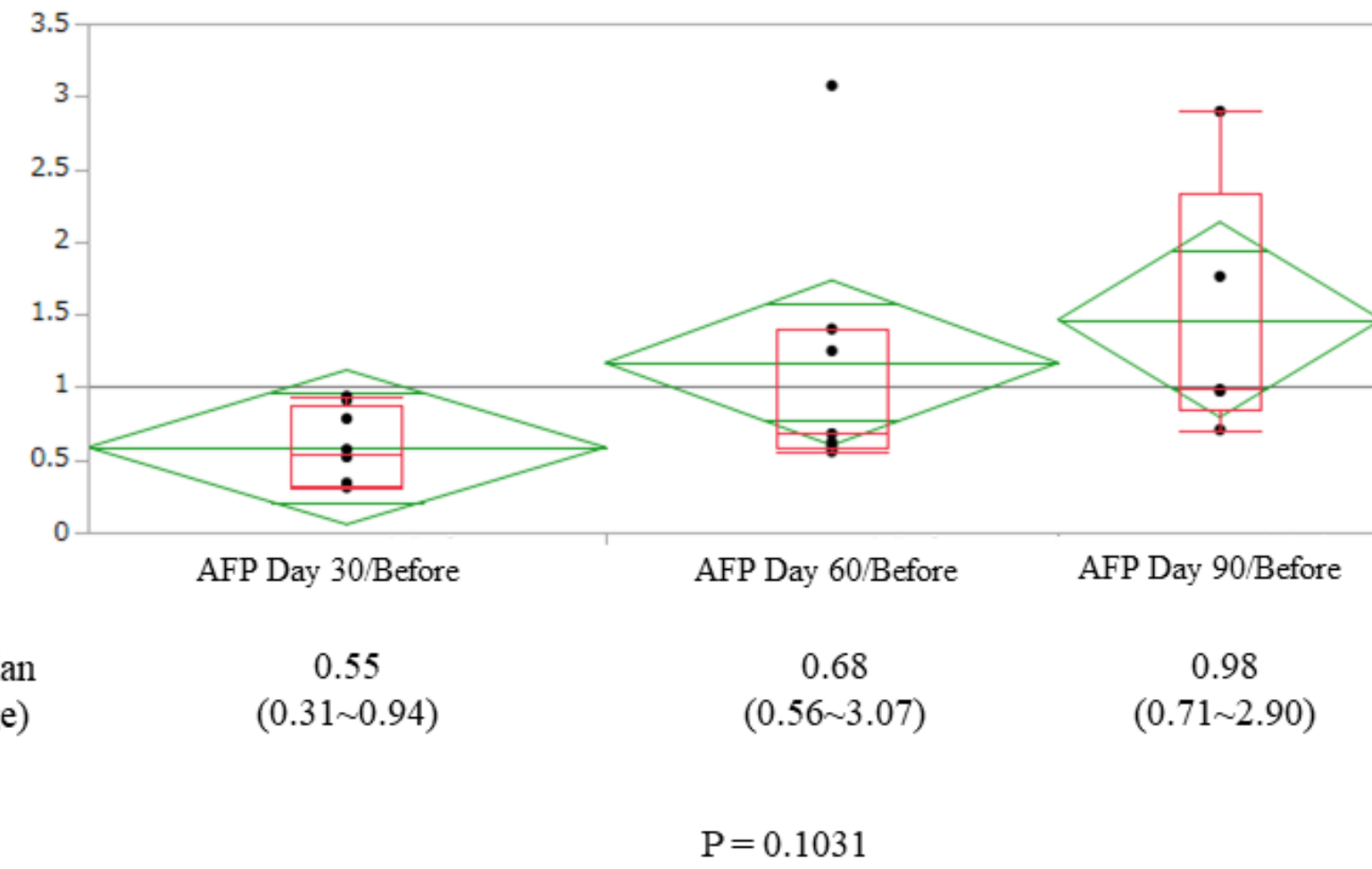

Figure 3

The proportions of the a-fetoprotein level at 1,2, and 3 months relative to the baseline level in patients receiving lenvatinib after atezolizumab plus bevacizumab therapy. 


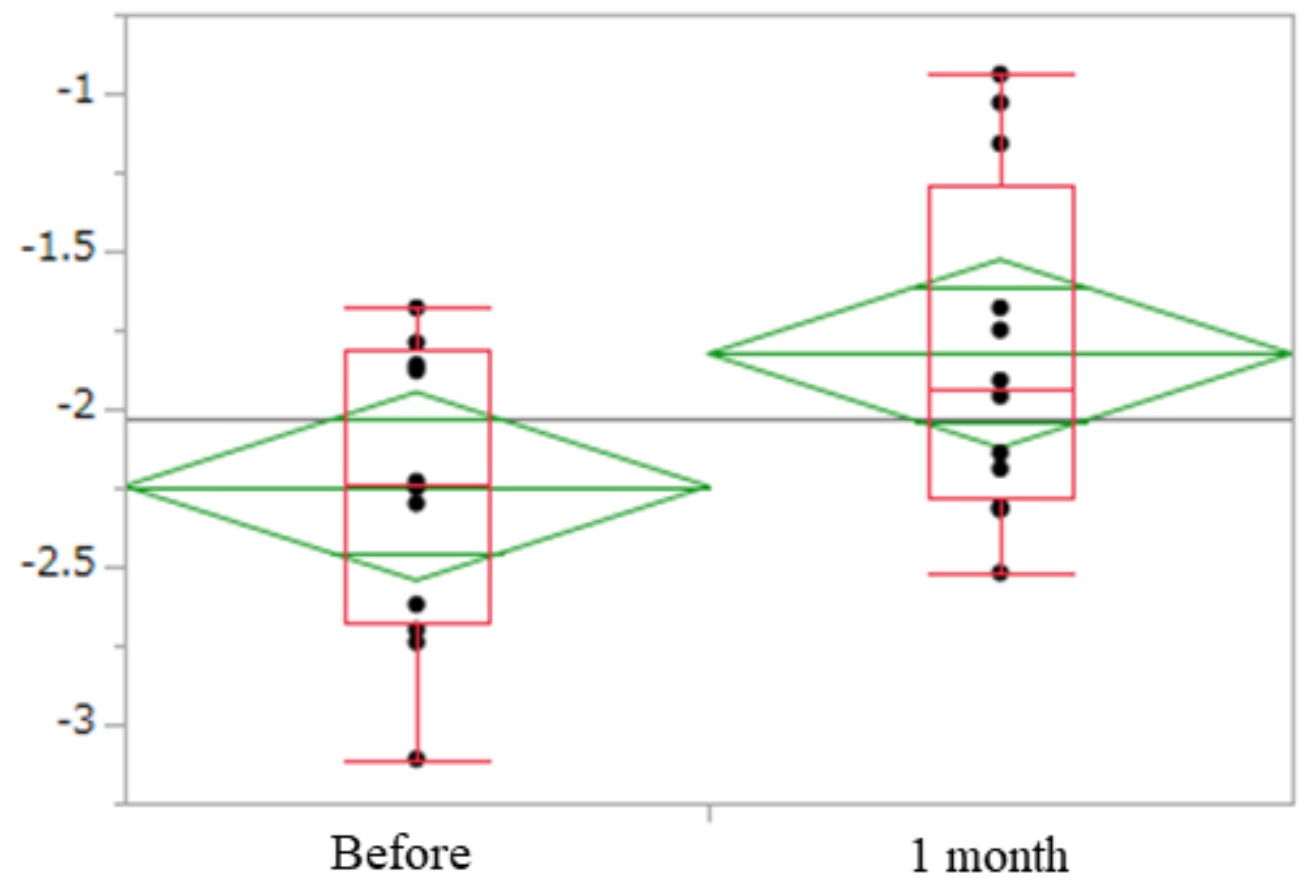

Median

$-2.24$

$(-3.11 \sim-1.68)$

$-1.94$

(range)

$(-2.52 \sim-0.94)$

$$
\mathrm{P}=0.0510
$$

\section{Figure 4}

The changes in ALBI score before and at 1 month in patients receiving lenvatinib after atezolizumab plus bevacizumab therapy. ALBI, albumin-bilirubin.

\section{Supplementary Files}

This is a list of supplementary files associated with this preprint. Click to download.

- SupplementaryFigure1.tif 